\title{
miRNA arm selection and isomiR distribution in gastric cancer
}

\author{
Sung-Chou Li ${ }^{1,2}$, Yu-Lun Liao ${ }^{3}$, Meng-Ru Ho ${ }^{1}$, Kuo-Wang Tsai ${ }^{4}$, Chun-Hung Lai ${ }^{3}$, Wen-chang Lin ${ }^{2,3^{*}}$ \\ From The Tenth Asia Pacific Bioinformatics Conference (APBC 2012) \\ Melbourne, Australia. 17-19 January 2012
}

\begin{abstract}
Background: MicroRNAs (miRNAs) are small non-protein-coding RNAs. miRNA genes need several biogenesis steps to form function miRNAs. However, the precise mechanism and biology involved in the mature miRNA molecules are not clearly investigated. In this study, we conducted in-depth analyses to examine the arm selection and isomiRs using NGS platform.

Methods: We sequenced small RNAs from one pair of normal and gastric tumor tissues with Solexa platform. By analyzing the NGS data, we quantified the expression profiles of miRNAs and isomiRs in gastric tissues. Then, we measured the expression ratios of $5 p$ arm to $3 p$ arm of the same pre-miRNAs. And, we used Kolmogorov-Smirnov (KS) test to examine isomiR pattern difference between tissues.

Results: Our result showed the $5 p$ arm and $3 p$ arm miRNA derived from the same pre-miRNAs have different tissue expression preference, one preferred normal tissue and the other preferred tumor tissue, which strongly implied that there could be other mechanism controlling mature miRNA selection in addition to the known hydrogen-bonding selection rule. Furthermore, by using the KS test, we demonstrated that some isomiR types preferentially occur in normal gastric tissue but other types prefer tumor gastric tissue.

Conclusions: Arm selections and isomiR patterns are significantly varied in human cancers by using deep sequencing NGS data. Our results provided a novel research topic in miRNA regulation study. With advanced bioinformatics and molecular biology studies, more robust conclusions and insight into miRNA regulation can be achieved in the near future.
\end{abstract}

\section{Background}

MicroRNAs (miRNAs) are small non-protein-coding RNAs. Their final functional products are RNA molecules rather than proteins. Although the functional products are different, like many protein-coding genes, miRNA genes also need several maturation steps to form the functional products, single-strand RNAs with approximately $22 \mathrm{nt}$. in length. After miRNA genes are transcribed, the full-length transcripts (pri-miRNAs) form a hairpin structure (pre-miRNA) plus two unpaired tails, which are trimmed out by Drosha. The premiRNA, composed of $5 p$ arm, $3 p$ arm and terminal

\footnotetext{
* Correspondence: wenlin@ibms.sinica.edu.tw

${ }^{2}$ Institute of Biomedical Informatics, National Yang-Ming University, Taipei, Taiwan

Full list of author information is available at the end of the article
}

loop, is further processed by Dicer, trimming out the terminal loop and releasing the miRNA/miRNA* duplex. The miRNA/miRNA* duplex is subsequently processed by RISC, which unwinds the miRNA/miRNA* duplex at the end with weaker hydrogen binding. So, the strand with free $5^{\prime}$ end is selectively included into RISC and served as mature miRNA [1,2].

Although mature miRNAs are derived from full-length transcripts of miRNA genes, the expression of miRNA genes does not guarantee the expression of mature miRNA. In other words, not all of the pri-miRNAs are processed into mature miRNAs [3-5]. This unequal maturation control comes from several regulatory steps. First, Drosha and Dicer have higher affinity to the primiRNAs and pre-miRNAs, respectively, whose terminal loops are moderate in size [6,7]. Furthermore, longer
C Biomed Central

(c) 2012 Li et al.; licensee BioMed Central Ltd. This is an open access article distributed under the terms of the Creative Commons Attribution License (http://creativecommons.org/licenses/by/2.0), which permits unrestricted use, distribution, and reproduction in any medium, provided the original work is properly cited. 
stem ( $33 \mathrm{bp})$ of pri-miRNA is preferred by Drosha [6]. Second, owing to the hydrogen-bonding selection mechanism, the $5 \mathrm{p}$ arm and $3 \mathrm{p}$ arm of the same premiRNA usually have unequal likelihoods to be selected as mature miRNAs [8].

Up to now, this hydrogen-bonding-based selection rule seems to be the major view point. However, recent studies brought new concepts that challenged the traditional miRNA maturation mechanism. First, previous studies showed that the orthologous pre-miRNAs, although highly similar with each other, preferred the $5 p$ arm in one species but the $3 p$ arm in another species $[9,10]$. This result challenged the hydrogen-bonding selection rule, implying that there could be other regulation mechanism controlling the $5 \mathrm{p}$ arm or $3 p$ arm selection. Second, with the application of NGS technology, mature miRNAs were often observed to present as different isoforms, named isomiRs [9,11,12]. Further analysis has implied that different isomiRs may contribute to regulations in Drosophila development [13].

In this study, we conducted in-depth analyses on these issues by using NGS technology to quantify the expression profiles of miRNAs and isomiRs in human gastric tissues. By measuring the expression ratios of $5 p$ arm to $3 p$ arm between tissues, we showed the $5 p$ arm and $3 p$ arm miRNA derived from the same pre-miRNAs have different tissue expression preference, one preferred normal tissue and the other preferred tumor tissue, which strongly implied that there could be other mechanism controlling arm selection in addition to the hydrogenbonding selection rule. Furthermore, by using the Kolmogorov-Smirnov statistics test, we demonstrated that some isomiR types preferentially occur in normal gastric tissue but other types might prefer tumor gastric tissue.

\section{Methods}

\section{Collecting sequence reads}

We applied Illumina (Solexa) platform for small RNA sequencing. One pair of normal gastric tissue (G1245N) and gastric tumor tissue (G1245T) were lysed with TissueLyser (Qiagen), followed by RNA extraction with TRIzol reagent (Invitrogen) according to the manufacturer's protocol. Then, the RNA samples were processed and sequenced. The generated sequence reads were processed to remove the 3' end adapter, if applicable. Only the clean reads, reads with adapter detected and trimmed, were used for analysis. Besides, considering the length distribution of mature miRNAs, we selected only the clean reads with length 18 to 25 nucleotides for analysis.

The initially analyzed normal and tumor data sets are not equal in size (19.7 million reads in G1245N and 26.0 million reads in $\mathrm{G} 1245 \mathrm{~T}$ ). Therefore, we tried to normalize them with a regression model. As a result, we got the equation $y=1.3004 x-0.1457$, where $\mathrm{x}$ and $\mathrm{y}$ denote the expression levels of miRNAs in G1245N and G1245T library, respectively. After this normalization procedure and plotted in a scatter plot, most of the data points distributed near the line with the slope of 0.9874 and the $\mathrm{R}^{2}$ value was 0.8831 . This result showed that the expression levels of most miRNAs did not vary between tissues and the miRNA expression data from the two the libraries was comparable.

\section{Mapping criteria}

The clean reads were grouped into unique clean reads, followed by tabulating the count of each unique clean read. For higher confidence, only the unique reads with read count equal to or larger than two were used for mapping back to human pre-miRNAs (miRBase 16). In order to eliminate ambiguous mapped loci caused by the high similarity between human paralogous mature miRNAs, such as hsa-miR-548a and hsa-miR-548b, we allowed no mismatch at the mapping procedure. Previous reports observed nucleotide additions at the 3' end of miRNAs [12,15-18], which may cause mismatches at the mapping procedure. Therefore, using FernandezValverde's strategy [13], we trimmed the last 3' end mismatch one by one until the mapping perfect-match reads are at least 18 nucleotides in length.

\section{Excluding random matches}

With the application of NGS technology, miRNA are reported to exist as isomiRs $[9,11,12]$. As shown in Additional file 1, the isomiRs (the red line alignments) shift from their corresponding miRBase reference miRNAs (dark and light blue bars) in terms of location. When sequence reads were mapped back to mature miRNAs, the alignment shift may result in mismatches. Therefore, in addition to the perfect match constraint, we adopted an alternative procedure. In order to exclude random match, the difference in start position between mature miRNA and mapped reads must be equal to or less than two nucleotides. While, the difference in end position between mature miRNA and mapped reads must be equal to or less than five nucleotides.

\section{Experimental validation of miRNA with stem-loop RT-PCR} In this study, we used stem-loop RT-PCR to validate the 5p arm miRNA of hsa-mir-1307 as described previously [20]. The RT primer (CTCAACTGGTGTCGTGGAGTCGGCAATTCAGTTGAGagccgg) contains a stem-loop sequence and a 6-nt overhang sequence resulting in the binding specificity to mature miRNA. For each RT reaction, $1 \mathrm{~g}$ of total RNA was converted into cDNA (miRNA-specific stem-loop RT, 2 nM, 500M dNTP and 0.5l Superscript III, Invitrogen, Carlsbad, CA) 
and was performed as follows: $16^{\circ} \mathrm{C}$ for $30 \mathrm{~min}$, followed by 50 cycles at $20{ }^{\circ} \mathrm{C}$ for $30 \mathrm{~s}, 42{ }^{\circ} \mathrm{C}$ for $30 \mathrm{~s}$ and $50{ }^{\circ} \mathrm{C}$ for $1 \mathrm{~s}$. Expression of the miRNA was detected with real-time quantitative PCR (RT-qPCR) by the SYBR Green I protocol (Applied Biosystems, Foster City, CA), $200 \mathrm{nM}$ miRNA-specific forward primer (CGGCGG tcgaccggacctcgac), and $200 \mathrm{nM}$ universal reverse primer. RT-qPCR was performed with the following conditions: $95{ }^{\circ} \mathrm{C}$ for $10 \mathrm{~min}, 95{ }^{\circ} \mathrm{C}$ for $15 \mathrm{~s}$ and $63{ }^{\circ} \mathrm{C}$ for $32 \mathrm{~s}$ by 40 cycles. All values were normalized against U6 RNA.

\section{Comparing isomiR patterns between tissues}

In this study, we applied the Kolmogorov-Smirnov (KS) test for determining whether isomiR distribution patterns differ between normal and tumor tissue. KS test tries to determine if two datasets differ significantly and has the advantage of making no assumption about the distribution of data. For each mature miRNA, we enumerated its isomiRs in normal and tumor tissue (Figure 1 ), forming a union, followed by summarizing the read counts of all isomiRs as the expression abundance of the miRNA. Then we assigned the isomiRs with a type
ID according to their locations. Next, we generated a table of relative abundance percentage of each isomiR type. By using this table, we may transform the $\times$ axis of KS test from numerical data into categorical data (isomiR type). As a result, we may examine individual isomiR type and whether the isomiR distributions are different between normal and tumor tissue according to the generated p-value of KS test.

\section{Results and discussion Summary of sequence reads}

In this study, we generated sequence reads of small RNAs from normal gastric tissue $(\mathrm{G} 1245 \mathrm{~N})$ and gastric tumor tissue (G1245T). Totally, 32.1 and 32.4million sequence reads were initially collected from G1245N and G1245T library, respectively. After trimming adapter procedure, we collected 23.8 and 29.9 million reads individually in G1245N and G1245T library for further analysis. Further filtered with length and read count criteria, in G1245N and G1245T library, 19.7 and 26.0 million reads were finally used to quantify miRNA expression level.

$$
\begin{aligned}
& \text { >hsa-mir-101-1 mi:11-32;MA: 47-67 } \\
& \text { ugc C CuggcuCAGUUAUCACAGUGCUGAUGCUgucuauucuaaaggUACAGUACUGUGAUAACUGAAggauggca } \\
& \cdot(((\ldots \ldots(((()(((()((()(((((.(1((\ldots \ldots \ldots \ldots)))))))))))))))))))))) \ldots \ldots))) \text {. }
\end{aligned}
$$

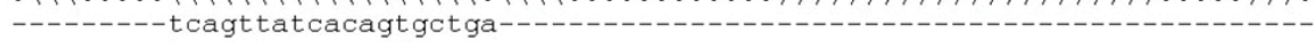

$\begin{array}{ll}3 & -1,-4 \\ 7 & -1,-3 \\ 374 & -1,-2 \\ 43 & -1,-1 \\ 12 & -1,0 \\ 10 & 0,-2 \\ 17 & 0,-1 \\ 7 & 0,0 \\ 2 & 0,1 \\ 5 & 1,1 \\ 6 & -2,-2 \\ 16 & -2,-1 \\ 321 & -1,-4 \\ 10936 & -1,-3 \\ 8349 & -1,-2 \\ 40495 & -1,-1 \\ 13316 & -1,0 \\ 715 & -1,1 \\ 1132 & 0,-3 \\ 4835 & 0,-2 \\ 51920 & 0,-1 \\ 93006 & 0,0 \\ 124873 & 0,1 \\ 254 & 0,2 \\ 26 & 0,3 \\ 19 & 1,-2 \\ 96 & 1,-1 \\ 308 & 1,0 \\ 482 & 1,1 \\ 69 & 2,-1 \\ 430 & 2,0 \\ 164 & 2,1 \\ & \end{array}$

Figure 1 Mapping result of hsa-mir-101-1 in G1245N library. hsa-mir-101-1 encode minor miRNA (hsa-miR-101*) at the 5p arm, ranging from nt. 11 to 32 of pre-miRNA, and major miRNA (hsa-miR-101) at the $3 p$ arm, ranging from nt. 47 to 67 . The $5 p$ and 3p miRNAs are presented in uppercase and they individually have 10 and 22 isomiR types, each of which is labeled with read count (before normalization) and location offset. The expression level of a mature miRNA is determined by summing the read counts of all its isomiRs. 


\section{Analysis of detected miRNAs}

Under our mapping criteria described in Methods, totally there were $17,492,248$ and $23,974,839$ reads, individually from G1245N and G1245T library, mapped to known human miRNAs (Table 1). Therefore, 88.8\% reads in G1245N library belong to miRNA; while, $92.4 \%$ reads in G1245T library do so. As mentioned in the previous study [12], the rest non-miRNA reads could be other small non-protein-coding RNAs or degradation product of mRNA. As shown in Table 1, 688 and 705 out of 1,223 known mature miRNAs were detected in G1245N and G1245T library, respectively. Our data is comparable to most NGS studies.

We arranged the miRNA reads within the mapped pre-miRNAs. As shown in Figure 1, hsa-mir-101-1 encodes mature miRNAs at both arms. The integer digits in middle column denote the read count of each isomiR. The presentations in the right column denote the location offset relative to reference miRNA annotated with miRBase. So, the reads with presentation " 0,0 " are exactly the same with reference miRNAs. Examining the counts of all reads, it is not guaranteed that the reference miRNAs from miRBase are the most abundant ones, which was also observed by other studies $[11,12,14]$. The mapping result of all pre-miRNAs in G1245N and G1245T library can be accessed in Additional file 2 and 3.

\section{Detection of additional miRNAs at the opposite arms}

Among 1,048 reported human pre-miRNAs, 730 were reported to encode mature miRNAs at only one arm (miRBase 16 release). However, with the improvement in sequencing depth of NGS platforms, more miRNAs can be also detected at the opposite arms. As illustrated in Additional file 2, hsa-mir-1307 encodes mature miRNA at only its $3 p$ arm by miRBase. However, we detected additional mature miRNA at the opposite arm,

Table 1 Summary of analysis on miRNA reads

\begin{tabular}{|c|c|c|}
\hline & G1245N & G1245T \\
\hline \# Reads & $17,492,248$ & $23,974,839$ \\
\hline \# detected pre-miRNAs ${ }^{a}$ & 542 & 556 \\
\hline$\#$ detected miRNAs ${ }^{a}$ & 688 & 705 \\
\hline \# miRNAs at opposite arm $^{b}$ & 87 & 96 \\
\hline \# isomiRs & 5,132 & 5,305 \\
\hline \# isomiRs with length equal to miRNA & 542 & 569 \\
\hline
\end{tabular}

We mapped the sequence reads back to human pre-miRNA, quantifying the expression levels of pre-miRNAs, mature miRNA and isomiRs.

a: The human pre-miRNA and mature miRNA are based on the definition of miRBase 16 . Totally, there are 1,048 pre-miRNAs and 1,223 mature miRNAs, resulting in 1,366 miRNA/pre-miRNA pairs.

b. According to miRBase 16,730 out of 1,048 human pre-miRNAs encode only one mature miRNA at one arm, either $5 p$ or $3 p$ arm. With the improvement on NGS's sequencing intensity, we detected additional opposite-arm mature miRNAs at the pre-miRNAs originally encoding only one miRNA. the $5 p$ arm, of hsa-mir-1307. In order to confirm the existence of the 5p miRNA of hsa-mir-1307, we designed specific stem-loop RT-PCR assay for validation. At the 5p arm of hsa-mir-1307, eight isomiRs were detected and we designed specific primers to validate the most abundant one. As shown in Figure 2, we can specifically detect the $5 \mathrm{p}$ arm miRNA of hsa-mir- 1307 in both normal and tumor tissue.

As described in previous study [14], the additional opposite-arm miRNAs are not necessary to be at lower expression levels than the original ones. Many of the additional opposite-arm miRNAs have higher expression level than the original one (Additional file 2 and 3), which might be different from the previous nomenclature rules. In this study, we totally detected 24 additional opposite-arm miRNAs exclusively in G1245N, 33 ones exclusively in G1245T and 63 ones both in G1245N and G1245T library. The discovery of additional opposite-arm miRNAs is because we mapped the sequence reads back to pre-miRNAs rather than only to mature miRNAs. Our study here provides a new way to further interrogate the miRNA/isomiR expression by carefully examining NGS data.

\section{Analysis of addition fragments generated by $3^{\prime}$ end modification}

Owing to 3' end modification [12,15-18], the altered nucleotides at the 3' end of reads may cause mismatches at the mapping procedure, making the originally perfect match reads fail to be mapped back to miRNAs. Therefore, we trimmed the terminal 3' end mismatch one nucleotide by one nucleotide, followed by analyzing the trimmed fragments. As a result, 2,766,852 reads in G1245N tissue and 3,319,704 reads in G1245T tissue were found to have nucleotide added at their 3' ends, individually accounting for $14.0 \%$ and $12.8 \%$ of read collection used for mapping. Without this alternative mapping method, these $14.0 \%$ and $12.8 \%$ more sequence reads can not be mapped back to human pre-miRNAs, which demonstrates the effectiveness of our alternative mapping procedure.

Previous large-scale investigation on Arabidopsis thaliana and Oryza sativa demonstrated uridine additions at the 3' end of miRNAs [17]. In this study, we totally observed 499 and 409 kinds of 3' end addition fragments in G1245N and G1245T library, respectively. In addition to uridine addition, we also observed adenine additions. As shown in Table 2, the most and the secondary abundant addition event in G1245N library is adenine and uridine addition, respectively. However, in G1245T library, the two addition events exchanged in terms of abundance. It needs more data to conclude whether such difference is functionally related to biological difference. In summary, adenine and uridine 


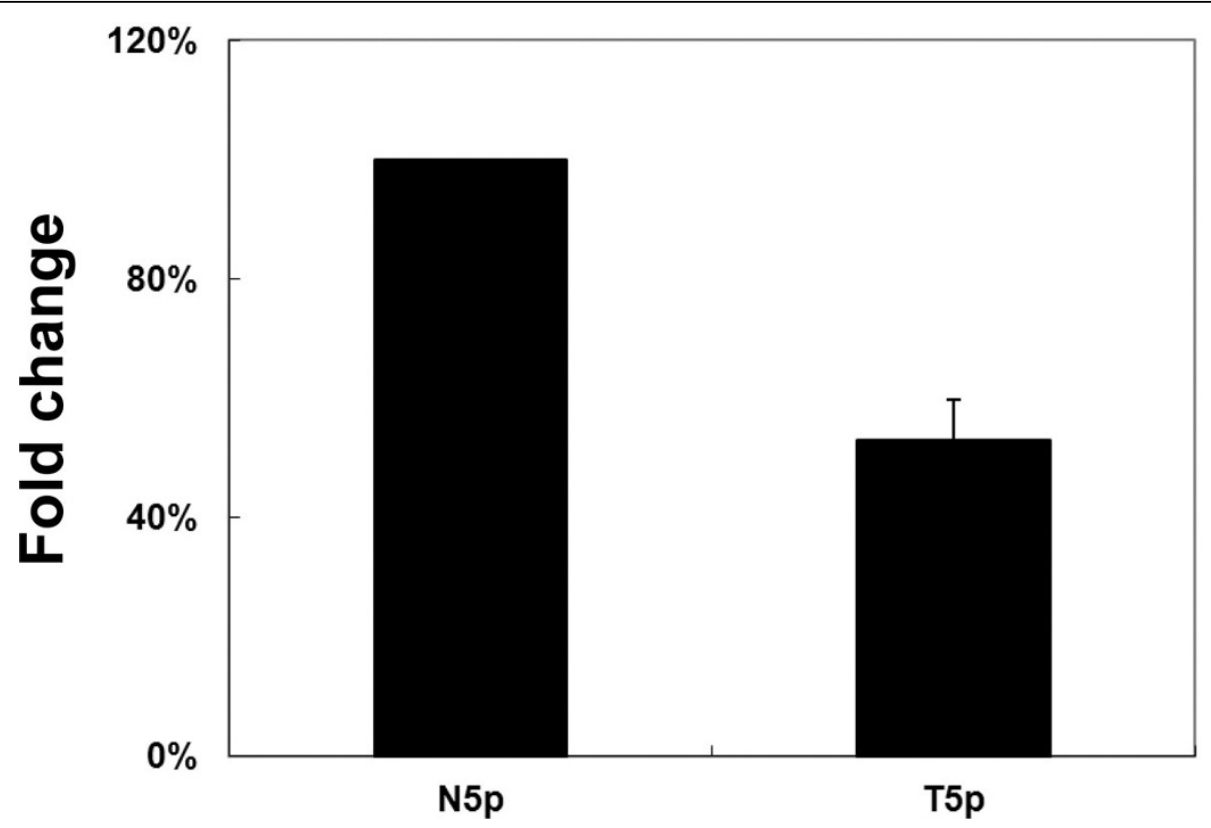

Figure 2 Detection of 5p arm miRNA of hsa-mir-1307. According to miRBase annotation, hsa-mir-1307 encodes mature miRNA at only its $3 p$ arm. We used stem-loop RT-PCR to succeed in detecting the additional opposite-arm miRNA in both G1245N and G1245T tissue.

additions account for almost $80 \%$ of all addition events. Besides, AA, UA, UU, AU, G and AAA also account for at least $1 \%$ of addition events.

Sequence variations at the 3' ends have been often observed in miRNA reads. Previous studies also reported several types of RNA editings, such as A to G transition catalyzed by adenosine deaminase and $\mathrm{C}$ to $\mathrm{U}$ transition catalyzed by cytidine deaminase [12,19], responsible for generating such variations. Owing to the existence of iso$\mathrm{miR}$, it is difficult to distinguish whether nucleotide addition or nucleotide modification contributes to such variations. As illustrated in Additional file 4, the terminal nucleotide variation, also called mismatch, could be generated from nucleotide modification from $\mathrm{C}$ to $\mathrm{U}$ at the terminus of the sequence read with 22 nucleotides,

Table 2 Distribution of the $3^{\prime}$ end addition fragments in G1245N and G1245T library

\begin{tabular}{ccc}
\hline Fragment sequence & Abun. in G1245N (\%) & Abun. in G1245T (\%) \\
\hline A & 50.93 & 33.25 \\
U & 30.72 & 44.07 \\
AA & 3.39 & 4.67 \\
UA & 2.28 & 4.19 \\
UU & 2.16 & 3.12 \\
AU & 1.53 & 1.23 \\
G & 1.40 & 1.80 \\
AAA & 1.07 & 2.10 \\
\hline
\end{tabular}

We analyzed the sequence and the relative abundance of $3^{\prime}$ end addition fragments. As a result, adenine and uridine additions account for almost $80 \%$ of all addition events. Only the addition fragments accounting for more than $1 \%$ of all addition events are listed here. altering the length of the read. Alternatively, it could also be generated from nucleotide addition of $U$ to the terminus of the read with 21 nucleotides, lengthening the read by one nucleotide. Additional molecular studies would be required to elucidate specific mechanism involved.

\section{Inconsistent expression ratios of $5 p$ arm to $3 p$ arm}

According to the hydrogen-bonding theory, the selection preference between pre-miRNA's $5 p$ arm and 3p arm is an intrinsic characteristic of pre-miRNA. However, if this selection theory is the only criterion deciding arm selection preference, the expression ratio of $5 p$ arm to $3 \mathrm{p}$ arm should be consistent, with only slight difference, wherever the sequencing samples come from. In order to examine whether the expression ratio for each premiRNA is consistent or not, we investigated its expression ratios of $5 \mathrm{p}$ arm to $3 \mathrm{p}$ arm in G1245N library and in G1245T library, naming them Ratio_N53p and Ratio_T53p, respectively. As shown in Table 3, the 5p and $3 \mathrm{p}$ miRNA of hsa-mir-423 individually have read counts of 20,920 (N5p) and 8,945 (N3p) in G1245N library, leading to the Ratio_N53p value of 2.3387. However, the Ratio_T53p value in G1245T library is 0.2726 , leading to 8.58 fold change between Ratio_N53p to Ratio_T53p. In order to avoid dramatic change in expression ratio of $5 p$ arm to $3 p$ arm, we examined only 166 pre-miRNAs whose N5p, N3p, T5p and T3p are equal to or larger than 10.

By an empirical criterion of fold change difference larger than three, 36 out of 166 examined pre-miRNAs 
Table 3 Inconsistent expression ratios of $5 p$ arm miRNA to $3 p$ arm miRNA between tissues

\begin{tabular}{ccccccccc}
\hline Pre-miRNA & Location & N5p & N3p & Ratio_N53p & T5p & T3p & Ratio_T53p & Fold change \\
\hline hsa-mir-17 & MA:14-36;mi:51-72 & 4,767 & 2,266 & 2.1037 & 6,656 & 425 & 15.6612 & 7.44 \\
hsa-mir-511-1 & 5p:16-36 & 39 & 903 & 0.0432 & 53 & 147 & 0.3605 & 8.35 \\
hsa-mir-511-2 & 5p:16-36 & 39 & 903 & 0.0432 & 53 & 147 & 0.3605 & 8.35 \\
hsa-mir-423 & 5p:17-39;3p:53-75 & 20,920 & 8,945 & 2.3387 & 1,708 & 6,266 & 0.2726 & 8.58 \\
hsa-mir-30c-1 & MA:17-39;mi:56-77 & 67,040 & 50 & $1,340.8000$ & 8,685 & 64 & 135.7031 & 9.88 \\
hsa-mir-135b & MA:16-38;mi:55-76 & 360 & 19 & 18.9474 & 171 & 99 & 1.7273 & 10.97 \\
hsa-mir-376a-1 & mi:7-28;MA:44-64 & 1,646 & 14 & 117.5714 & 569 & 54 & 10.5370 & 11.16 \\
hsa-mir-376a-2 & 3p:50-70 & 428 & 14 & 30.5714 & 99 & 54 & 1.8333 & 16.68 \\
hsa-mir-335 & MA:16-38;mi:52-73 & 3,509 & 110 & 31.9000 & 418 & 264 & 1.5833 & 20.15 \\
hsa-mir-30b & MA:17-38;mi:55-76 & 50,988 & 51 & 999.7647 & 3,931 & 89 & 44.1685 & 22.64 \\
\hline
\end{tabular}

Location column denotes the relative locations of mature miRNAs within their precursors. N5p, T5p individually denote the expression levels of $5 p$ arm miRNA (determined by summing the read counts of all its isomiRs) in normal and tumor tissue; while, N3p, T3p denote the corresponding information of 3p arm miRNA. The N5p and N3p values shown in this study are normalized.

show different expression ratios of $5 p$ to $3 p$ in normal and tumor gastric tissue. As shown in Figure 3, several pre-miRNAs have significantly different ratios, demonstrating that the arm selection preferences within the pre-miRNAs are significantly different between gastric normal and gastric tumor tissue. For more detailed examination, we listed the information of the 36 significant pre-miRNAs in Table 3 and Additional file 5. As illustrated in Table 3, the fold change can even reach up to more than 20. Among the significant altered pre-miRNAs, hsa-mir-17 is a very interesting case, at which $5 p$ arm has almost two times higher expression level than $3 p$ arm in normal tissue. However, in tumor tissue, the $5 p$ arm becomes about 1.4 times higher than in normal tissue but the $3 \mathrm{p}$ arm becomes 5.3 times lower, which results in the large difference in expression ratio of $5 p$ to $3 p$. Even with large difference in expression ratio of $5 p$ to $3 \mathrm{p}$, the major arm of hsa-mir-17 in normal and tumor tissue is the same one, the $5 p$ arm. In another interesting case of hsa-mir-423, the major arm and minor arm in normal tissue are individually $5 p$ arm and $3 p$ arm, obeying the annotation of miRBase. However, in tumor tissue, the major arm and minor arm are individually the $3 p$ arm and $5 p$ arm, totally reverse to miRBase annotation. In this case, the preferential arm in normal and tumor tissue shift from $5 \mathrm{p}$ to $3 \mathrm{p}$.

In summary, our result showed the $5 p$ and $3 p$ preference is not always consistent between biological samples, implying there could be other regulation mechanisms, in addition to the hydrogen-bonding-based selection rule, controlling the selection of $5 p$ or $3 p$. If so, this regulation mechanism could play important roles in oncogenesis process. This is a novel area to study the relationship between miRNAs and cancers. Therefore, more efforts should be applied in the subsequent studies.






\section{Arm selection preference between normal and tumor tissue}

In our previous study, we have shown arm selection preference of the same pre-miRNAs could vary between species [9]. In this study, our result implies there could be regulation mechanism, in addition to the hydrogenbonding-based selection rule, controlling the selection of $5 \mathrm{p}$ arm or $3 \mathrm{p}$ arm between different tissues and samples. Therefore, we are curious about whether difference on arm selection preference can be observed between tissues, especially between normal and tumor tissue. We first examined whether the arm selection preference annotated by miRBase is consistent with our NGS expression data. As shown in Table 4, the arm selection preferences of 22 pre-miRNAs are opposite to miRBase annotation. According to miRBase annotation, hsa-mir1277, hsa-mir-376a-2, hsa-mir-495, hsa-mir-659, hsamir-1303, hsa-mir-1307 and so on encode mature miRNA at only their $3 \mathrm{p}$ arms. We not only detected mature miRNA at their $5 \mathrm{p}$ arms but also observed that the newly detected $5 \mathrm{p}$ arms have higher expression levels than the originally annotated $3 p$ arm. Besides, the newly detected 3p arms of hsa-mir-511-1, hsa-mir-5112, hsa-mir-1273c and hsa-mir-1247 also have higher expression levels than the originally annotated $5 \mathrm{p}$ arm.

For the pre-miRNAs originally annotated to encode miRNAs at both arms, the major arms of hsa-mir-374a, hsa-mir-500a, hsa-mir-625 and hsa-mir-136 are their 5p arms; while, the major arms of hsa-mir-664, hsa-mir144, hsa-mir-493 and hsa-mir-376a-1 are their 3p arms. According to NGS expression data, we observed that their major arms and minor arms expression levels reversed, leading to an observation different from miRBase annotation. Among them, hsa-mir-374a and hsamir-144 are two extreme cases, at which the miRBaseannotated minor arms individually have about 17 or 28 times as high expression levels as the miRBase-annotated major arms have. In summary, our result demonstrated that arm selection preference could vary. In order to solve this debate, more NGS expression data should be included and such phenomenon should be studied further.

Since arm selection preference is inconsistent between known reported miRNAs and our NGS expression data, we further investigated whether arm selection preference could vary between normal and tumor tissue. For this purpose, we examined whether arm selection preference differs between normal and tumor tissue. In other words, we investigated whether the $5 \mathrm{p}$ arm and $3 \mathrm{p}$ arm miRNA derived from the same pre-miRNA have reversal tissue expression preference without considering miRBase annotation. As shown in Table 5, we observed four additional pre-miRNAs possessing this property. Within hsa-mir-136, hsa-mir-423, hsa-mir-376b and hsa-mir-

Table 4 The pre-miRNAs whose arm selection preferences are not consistent with miRBase annotation

\begin{tabular}{|c|c|c|c|c|c|c|}
\hline pre-miRNA & Location & N5p & N3p & T5p & T3p & MAmiExch \\
\hline hsa-mir-1277 & $3 p: 47-68$ & 354 & 172 & 201 & 56 & $\mathrm{~N} ; \mathrm{T}$ \\
\hline hsa-mir-376a-2 & 3p:50-70 & 428 & 14 & 99 & 54 & $\mathrm{~N} ; \mathrm{T}$ \\
\hline hsa-mir-495 & 3p:50-71 & 35 & 19 & 12 & 5 & $\mathrm{~N} ; \mathrm{T}$ \\
\hline hsa-mir-1303 & 3p:52-73 & 19 & 73 & 12 & 10 & $\mathrm{~T}$ \\
\hline hsa-mir-1306 & 3p:55-72 & 94 & 0 & 31 & 0 & $N ; T$ \\
\hline hsa-mir-496 & 3p:56-77 & 27 & 0 & 50 & 0 & $\mathrm{~N} ; \mathrm{T}$ \\
\hline hsa-mir-561 & 3p:61-82 & 231 & 0 & 40 & 0 & $\mathrm{~N} ; \mathrm{T}$ \\
\hline hsa-mir-659 & 3p:61-82 & 123 & 0 & 56 & 0 & $\mathrm{~N} ; \mathrm{T}$ \\
\hline hsa-mir-376b & $3 p: 62-83$ & 106 & 9 & 32 & 83 & $N$ \\
\hline hsa-mir-1307 & 3p:80-101 & 3512 & 2369 & 2002 & 3544 & $\mathrm{~N}$ \\
\hline hsa-mir-1273c & $5 p: 10-31$ & 0 & 53 & 0 & 50 & $N ; \mathrm{T}$ \\
\hline hsa-mir-511-1 & 5p:16-36 & 39 & 903 & 53 & 147 & $\mathrm{~N} ; \mathrm{T}$ \\
\hline hsa-mir-511-2 & 5p:16-36 & 39 & 903 & 53 & 147 & $\mathrm{~N} ; \mathrm{T}$ \\
\hline hsa-mir-1247 & $5 p: 40-61$ & 67 & 237 & 17 & 41 & $\mathrm{~N} ; \mathrm{T}$ \\
\hline hsa-mir-374a & MA:12-33;mi:42-63 & 2275 & 36122 & 2017 & 35147 & $\mathrm{~N} ; \mathrm{T}$ \\
\hline hsa-mir-500a & MA:13-35;mi:52-73 & 215 & 1907 & 72 & 1834 & $\mathrm{~N} ; \mathrm{T}$ \\
\hline hsa-mir-625 & MA:15-35;mi:52-73 & 333 & 576 & 87 & 206 & $\mathrm{~N} ; \mathrm{T}$ \\
\hline hsa-mir-136 & MA:15-37;mi:49-70 & 1328 & 621 & 530 & 652 & $\mathrm{~T}$ \\
\hline hsa-mir-664 & mi:11-34;MA:49-71 & 913 & 271 & 398 & 98 & $\mathrm{~N} ; \mathrm{T}$ \\
\hline hsa-mir-144 & mi:15-36;MA:52-71 & 12406 & 441 & 1209 & 113 & $\mathrm{~N} ; \mathrm{T}$ \\
\hline hsa-mir-493 & mi:16-37;MA:57-78 & 254 & 248 & 1744 & 727 & $\mathrm{~N} ; \mathrm{T}$ \\
\hline hsa-mir-376a-1 & mi:7-28;MA:44-64 & 1646 & 14 & 569 & 54 & $\mathrm{~N} ; \mathrm{T}$ \\
\hline
\end{tabular}

$\mathrm{N}$ and T symbols in "MAmiExch" column represent the normal and tumor library in which such major and minor arm exchange event was observed. 
Table 5 Arm selection preference of $5 p$ arm and $3 p$ arm miRNA exchange between gastric normal and gastric tumor tissue

\begin{tabular}{cccccc}
\hline pre-miRNA & Location & N5p & N3p & T5p & T3p \\
\hline hsa-mir-136 & MA:15-37;mi:49-70 & 1328 & 621 & 530 & 652 \\
hsa-mir-361 & 5p:6-27;3p:45-67 & 1651 & 2493 & 1451 & 1024 \\
hsa-mir-423 & 5p:17-39;3p:53-75 & 20920 & 8945 & 1708 & 6266 \\
hsa-mir-376b & 3p:62-83 & 106 & 9 & 32 & 83 \\
hsa-mir-1307 & 3p:80-101 & 3512 & 2369 & 2002 & 3544 \\
\hline
\end{tabular}

In this table, the major and minor mature miRNA dereived from the same premiRNA exchange between gastric normal and gastric tumor tissue.

1307, their 5p arm miRNAs have higher expression level in the normal tissue; while their $3 \mathrm{p}$ arm miRNAs dominated in the tumor tissue. In the contrary, the $5 \mathrm{p}$ arm of hsa-mir-361 is the major miRNA in tumor tissue and the 3p arm miRNA is the major one in normal tissue.

Although derived from the same gene locus and transcribed by the same transcription factors, the $5 p$ arm and $3 p$ arm miRNA might have reversal expression preference. It is likely that such an observation is generated by NGS platform dependent biases. However, it is possible that there could be an unknown selection mechanism, during maturation procedure, controlling the arm selection preference between normal and tumor tissue. According to our data, this idea is reasonable and deserves more efforts to put it into examination.

\section{Systematic investigation on isomiR distribution between tissues}

Previous report showed that different isomiR types may contribute to different regulation in different tissues [13]. In this study, we are curious about whether isomiR distribution patterns are diverse between gastric normal and gastric tumor tissue, namely normal tissue prefers several specific isomiR types and tumor prefers the others. In order to solve this problem, we applied Kolmogorov-Smirnov (KS) test in determining significant difference on isomiR distribution patterns. KS test tries to determine if two datasets differ significantly under the null hypothesis that the samples are drawn from the same distribution. Although KS test has the advantage of making no assumption about the distribution of data, it is sensitive to the median. Besides, when a miRNA has significant low expression level, only few isomiR types can be presented, inducing biased isomiR distribution patterns. Therefore, we selected only the $169 \mathrm{miR}$ NAs with more than eight kinds of isomiR types and read counts more than 1000 in both normal and tumor tissue for comparison.

Under our p value criterion $<0.001$ (see Methods), 42 out of the 169 examined miRNAs are identified to have different isomiR distribution patterns between normal and tumor gastric tissue. The detailed information of the significant miRNAs is listed in Additional file 6 . Below, we illustrated several cases for discussion. As illustrated in Figure 4a, the expression levels of hsamiR-497 are approximately equal in both tissues; while, it has diverse isomiR distribution patterns ( $\mathrm{p}$ value $\approx 0$ ) between G1235N and G1245T tissue. In G1245N, the most and secondary abundant isomiRs are type four and type five. In G1245T, isomiR type four and type five reversed in terms of abundance. In Figure $4 \mathrm{~b}$, although the isomiR preferences of hsa-let-7a between tissues are the same, the most abundant isomiR type in G1245T is $20 \%$ less in G1245N. Moreover, both the secondary and tertiary abundant isomiR type in G1245N increase $10 \%$ than that in G1245T.

For the previous two cases, the expression levels between two tissues are almost equal. In Figure 4c, the expression levels of hsa-miR-21 are different between tissues, with fold change (fc) equal to 2.4051. In this case, the isomiR distribution patterns are diverse. The isomiR type peaks shift and both the most and secondary abundant isomiR type between tissues are different. So far, we have shown no matter the fc value is small or large, the isomiR distribution type can be diverse between tissues. Next, in Figure 4d, even the fc value of hsa-miR-30b reaches up to 12.97 , the isomiR distribution patterns are not diverse between tissues.

Although isomiRs are highly overlapped with each other, they could have difference at the 5' end, leading to alteration at the seed region. The complementary binding between miRNAs and target mRNAs mainly depends on the binding within the seed region. Therefore, the difference at the 5 'end between isomiRs is supposed to alter isomiRs' target genes. As a result, different isomiRs from the miRNAs could have different target genes, involved in different activities or pathways. Hence, it is reasonable that different isomiRs may contribute to various regulation pathways in different tissues. And, this type of isomiR regulation could have significant biological consequences.

\section{Conclusions}

In this study, we applied NGS data to quantify miRNA expression profiles between gastric normal and gastric tumor tissue. Our data showed that although derived from the same pre-miRNAs, $5 p$ arm miRNA and $3 p$ arm miRNA can have reversed expression preferences, implying there could be other regulation mechanism controlling $5 \mathrm{p}$ or $3 \mathrm{p}$ selection. Moreover, although derived from the same mature miRNA, isomiRs can have different expression preference, some prefer normal tissue and the other prefer tumor tissue. Although we examined only one pair of normal and tumor tissue, our results provided a novel research topic in miRNA regulation study. With more tissue samples examined, 

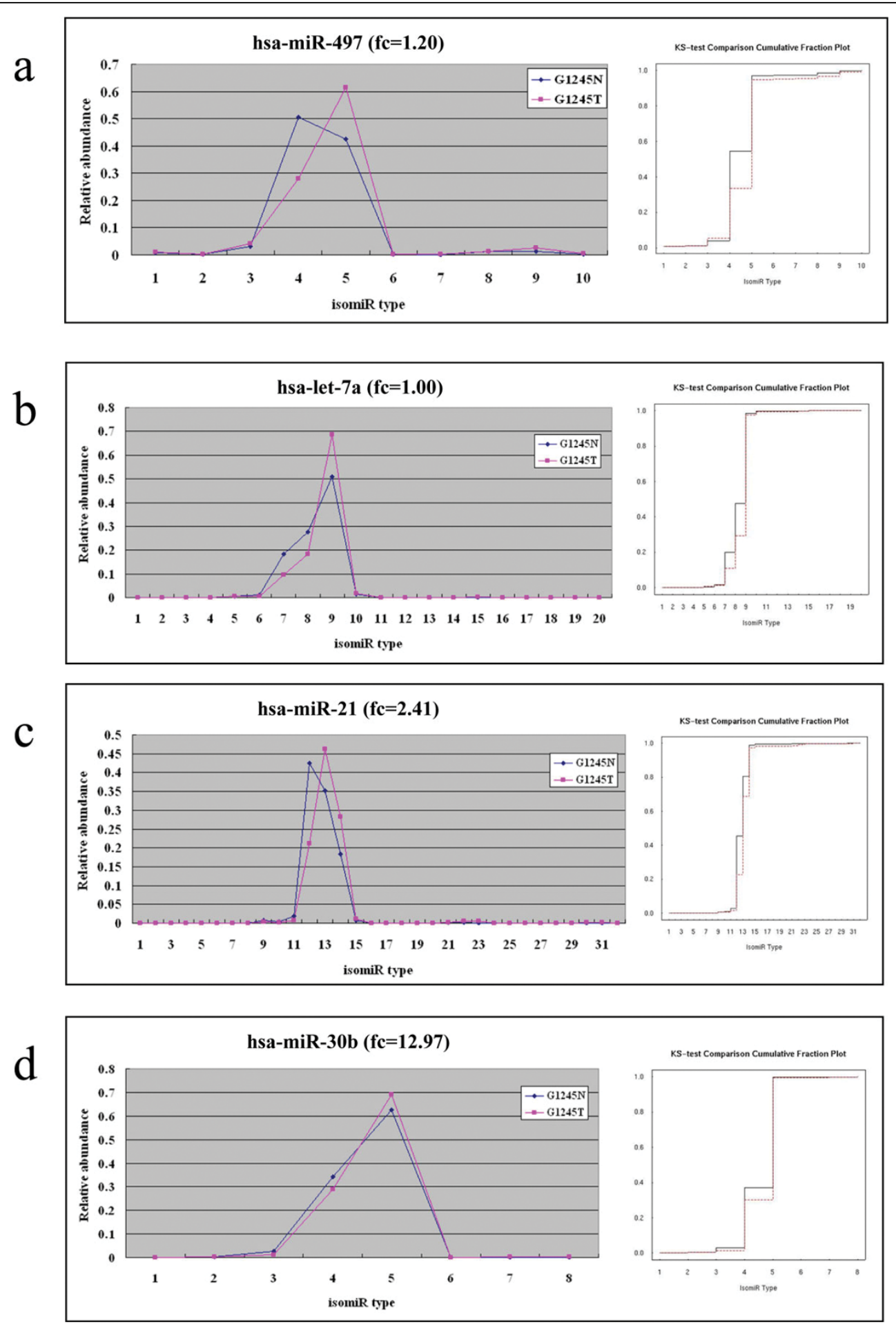

Figure 4 IsomiR distribution patterns mature miRNAs between G1245N and G1245T library. The isomiR distribution patterns (left panel) and the corresponding KS-test comparison plot (right panel) are provided. (a) The most abundant isomiRs in two tissues are type four and type five, respectively. (b) The most abundant isomiR type in G1245T is $20 \%$ less in G1245N. (c) The most abundant isomiRs in two tissues are individually type 12 and type 13. (d) Even expression levels of hsa-miR-30b are dramatically different between tissues, the isomiR distribution patterns are still the same. 
we can have more robust conclusions and perform the studies with insight into miRNA regulation.

\section{Additional material}

Additional file 1: The criteria of our mapping procedure. In order to exclude random match, the difference in start position between mature miRNA and mapped reads must be equal to or less than two. While, the difference in end position between mature miRNA and mapped reads must be equal to or less than five.

Additional file 2: The mapping result of all pre-miRNAs in G1245N library. We arranged the miRNA reads from G1245N library within the mapped pre-miRNAs. The read count and location offset of each isomiR are provided.

Additional file 3: The mapping result of all pre-miRNAs in G1245N library. We arranged the miRNA reads from G1245T library within the mapped pre-miRNAs. The read count and location offset of each isomiR are provided.

Additional file 4: The difficulty in distinguishing nucleotide addition from nucleotide modification. In this case, the last nucleotide variation could be generated from nucleotide modification from $\mathrm{C}$ to $\mathrm{U}$ at the terminus of the sequence read with 22 nucleotides, which does not alter the length of the read. However, it could also be generated from nucleotide addition of $\mathrm{U}$ to the terminus of the read with 21 nucleotides, which lengthens the read by one nucleotide.

Additional file 5: Inconsistent expression ratios of $5 p$ arm miRNA to $3 p$ arm miRNA. The expression ratios of $5 p$ arm miRNA to $3 p$ arm miRNA are not consistent between gastric normal and gastric tumor tissue, which implies the arm selection preference of $5 p$ arm and $3 p$ arm may varyn between tissues.

Additional file 6: List of miRNAs whose isomiR distribution patterns significantly differ between normal and tumor tissue. Normal and Tumor denote the expression level of mature miRNA in gastric normal and gastric tumor tissue, respectively. Fold change denote the fold change of miRNA expression level.

\section{Abbreviations}

microRNA, NGS, isomiR, gastric tumor, arm selection.

\section{Acknowledgements}

We thank Yourgene Bioscience http://www.yourgene.com.tw for providing the sequencing service. This work was supported by grants from Academic Sinica and National Science Council of Taiwan.

This article has been published as part of BMC Genomics Volume 13 Supplement 1, 2012: Selected articles from the Tenth Asia Pacific Bioinformatics Conference (APBC 2012). The full contents of the supplement are available online at http://www.biomedcentral.com/1471-2164/13? issue $=\mathrm{S} 1$.

\section{Author details}

'Genomics Research Center, Academia Sinica, Taipei, Taiwan. ${ }^{2}$ Institute of Biomedical Informatics, National Yang-Ming University, Taipei, Taiwan. ${ }^{3}$ Institute of Biomedical Sciences, Academia Sinica, Taipei, Taiwan.

${ }^{4}$ Department of Medical Education and Research, Kaohsiung Veterans General Hospital, Kaohsiung, Taiwan.

\section{Authors' contributions}

SCL executed this study and wrote the draft of this manuscript. YLL is responsible for $P C R$ validation of miRNA. MRH helped calculate the $p$ value of KS test. KWT and CHL helped tissue preparation and RNA extraction. WCL supervised the study and edited the manuscript.

\section{Competing interests}

The authors declare that they have no competing interests.
Published: 17 January 2012

\section{References}

1. Bartel DP: MicroRNAs: genomics, biogenesis, mechanism, and function. Cell 2004, 116(2):281-297.

2. Schwarz DS, Hutvagner G, Du T, Xu Z, Aronin N, Zamore PD: Asymmetry in the assembly of the RNAi enzyme complex. Cell 2003, 115(2):199-208.

3. Suzuki HI, Miyazono K: Emerging complexity of microRNA generation cascades. J Biochem 2011, 149(1):15-25.

4. Trabucchi M, Briata P, Filipowicz W, Rosenfeld MG, Ramos A, Gherzi R: How to control miRNA maturation? RNA Biol 2009, 6(5):536-540.

5. Tsai KW, Wu CW, Hu LY, Li SC, Liao YL, Lai CH, Kao HW, Fang WL, Huang KH, Chan WC, et al: Epigenetic regulation of miR-34b and miR-129 expression in gastric cancer. Int J Cancer 2011, 129:2600-2610.

6. Zeng Y, Yi R, Cullen BR: Recognition and cleavage of primary microRNA precursors by the nuclear processing enzyme Drosha. EMBO J 2005, 24(1):138-148.

7. Zhang $X$, Zeng $Y$ : The terminal loop region controls microRNA processing by Drosha and Dicer. Nucleic Acids Res 2010, 38(21):7689-7697.

8. Griffiths-Jones S, Grocock RJ, van Dongen S, Bateman A, Enright AJ: miRBase: microRNA sequences, targets and gene nomenclature. Nucleic Acids Res 2006, 34(Database issue):D140-D144.

9. Li SC, Chan WC, Ho MR, Tsai KW, Hu LY, Lai CH, Hsu CN, Hwang PP, Lin WC: Discovery and characterization of medaka miRNA genes by next generation sequencing platform. BMC Genomics 2010, 11(Suppl 4):S8.

10. Griffiths-Jones S, Hui JH, Marco A, Ronshaugen M: MicroRNA evolution by arm switching. EMBO Rep 2011, 12(2):172-177.

11. Glazov EA, Cottee PA, Barris WC, Moore RJ, Dalrymple BP, Tizard ML: A microRNA catalog of the developing chicken embryo identified by a deep sequencing approach. Genome Res 2008, 18(6):957-964.

12. Morin RD, O'Connor MD, Griffith M, Kuchenbauer F, Delaney A, Prabhu AL, Zhao Y, McDonald H, Zeng T, Hirst M, et al: Application of massively parallel sequencing to microRNA profiling and discovery in human embryonic stem cells. Genome Res 2008, 18(4):610-621.

13. Fernandez-Valverde SL, Taft RJ, Mattick JS: Dynamic isomiR regulation in Drosophila development. RNA 2010, 16(10):1881-1888.

14. Li SC, Chan WC, Lai CH, Tsai KW, Hsu CN, Jou YS, Chen HC, Chen $\mathrm{CH}$ Lin WC: UMARS: Un-MAppable Reads Solution. BMC Bioinformatics 2011, 12(Suppl 1):S9.

15. Siomi MC, Siomi H: Characterization of endogenous human Argonautes and their miRNA partners in RNA silencing. Nucleic Acids Symp Ser (Oxf) 2008, 52:59-60.

16. Landgraf $P$, Rusu M, Sheridan R, Sewer A, lovino N, Aravin A, Pfeffer S, Rice A, Kamphorst AO, Landthaler M, et al: A mammalian microRNA expression atlas based on small RNA library sequencing. Cell 2007, 129(7):1401-1414.

17. Ebhardt HA, Tsang HH, Dai DC, Liu Y, Bostan B, Fahlman RP: Meta-analysis of small RNA-sequencing errors reveals ubiquitous post-transcriptional RNA modifications. Nucleic Acids Res 2009, 37(8):2461-2470.

18. Reid JG, Nagaraja AK, Lynn FC, Drabek RB, Muzny DM, Shaw CA, Weiss MK, Naghavi AO, Khan M, Zhu H, et al: Mouse let-7 miRNA populations exhibit RNA editing that is constrained in the $5^{\prime}$-seed/cleavage/anchor regions and stabilize predicted mmu-let-7a:mRNA duplexes. Genome Res 2008, 18(10):1571-1581.

19. Wheeler BM, Heimberg AM, Moy VN, Sperling EA, Holstein TW, Heber S, Peterson KJ: The deep evolution of metazoan microRNAs. Evol Dev 2009, 11(1):50-68.

20. Chen SJ, Chen GH, Chen YH, Liu CY, Chang KP, Chang YS, Chen HC Characterization of Epstein-Barr virus miRNAome in nasopharyngeal carcinoma by deep sequencing. PLoS One 2010, 5(9):e12745.

doi:10.1186/1471-2164-13-S1-S13

Cite this article as: Li et al:: miRNA arm selection and isomiR distribution in gastric cancer. BMC Genomics 2012, 13(Suppl 1):S13. 